\title{
ON THE PRIME GRAPH OF A FINITE GROUP
}

\author{
M. GHORBANI, M. R. DARAFSHEH, AND PEDRAM YOUSEFZADEH
}

Received 12 May, 2015

\begin{abstract}
Let $G$ be a finite group. We define the prime graph $\Gamma(G)$ of $G$ as follows: The vertices of $\Gamma(G)$ are the primes dividing the order of $G$ and two distinct vertices $p, q$ are joined by an edge, denoted by $p \sim q$, if there is an element in $G$ of order $p q$. We denote by $\pi(G)$, the set of all prime divisors of $|G|$. The degree $\operatorname{deg}(p)$ of a vertex $p$ of $\Gamma(G)$ is the number of edges incident with $p$. If $\pi(G)=\left\{p_{1}, p_{2}, \ldots, p_{k}\right\}$ where $p_{1}<p_{2}<\ldots<p_{k}$, then we define $D(G)=\left(\operatorname{deg}\left(p_{1}\right), \operatorname{deg}\left(p_{2}\right), \ldots, \operatorname{deg}\left(p_{k}\right)\right)$, which is called the degree pattern of $G$. Given a finite group $M$, if the number of non-isomorphic groups $G$ such that $|G|=|M|$ and $D(G)=D(M)$ is equal to $r$, then $M$ is called $r$-fold OD-characterizable. Also a 1-fold OD-characterizable group is simply called OD-characterizable. In this paper we give some results on characterization of finite groups by prime graphs and OD-characterizability of finite groups. In particular we apply our results to show that the simple groups $G_{2}(7), B_{3}(5), \mathbb{A}_{11}$, and $\mathbb{A}_{19}$ are OD-characterizable.
\end{abstract}

2000 Mathematics Subject Classification: 20D05; 20D06; 20D60

Keywords: prime graph, finite group, degree pattern

\section{INTRODUCTION}

Throughout this paper, groups under consideration are finite. For any group $G$, we denote by $\pi(G)$ the set of prime divisors of $|G|$. We denote the set of elements of $G$ by $\pi_{e}(G)$. We associate to $\pi_{e}(G)$ a graph called prime graph of $G$, denoted by $\Gamma(G)$. The vertex set of this graph is $\pi(G)$ and two distinct vertices $p, q$ are joined by an edge, denote by $p \sim q$, if $p q \in \pi_{e}(G)$. The connected components of $\Gamma(G)$ is denoted by $\pi_{1}, \pi_{2}, \ldots, \pi_{t(G)}$, where $t(G)$ is the number of connected components of $\Gamma(G)$. If the order of $G$ is even, the notation is chosen so that $2 \in \pi_{1}$. Clearly the order of $G$ can be expressed as the product of $m_{1}, m_{2}, \ldots, m_{t(G)}$, where $\pi\left(m_{i}\right)=\pi_{i}, 1 \leq i \leq t(G)$.

The degree $\operatorname{deg}(p)$ of a vertex $p$ of $\Gamma(G)$ is the number of edges incident with $p$. If $\pi(G)=\left\{p_{1}, p_{2}, \ldots, p_{k}\right\}$ with $p_{1}<p_{2}<\ldots<p_{k}$, then we define

$$
D(G)=\left(\operatorname{deg}\left(p_{1}\right), \operatorname{deg}\left(p_{2}\right), \ldots, \operatorname{deg}\left(p_{k}\right)\right),
$$

which is called the degree pattern of $G$. Given a finite group $M$, if the number of nonisomorphic groups $G$ such that $|G|=|M|$ and $D(G)=D(M)$ is equal to $r$, then $M$ is called $r$-fold OD-characterizable. Also a 1-fold OD-characterizable group is simply called OD-characterizable. 
We call a directed graph strongly connected if there is a directed path from each vertex in the graph to every other vertex. Given an integer $a$ and a positive integer $n$ with $(a, n)=1$, the multiplicative order of $a$ modulo $n$ is the smallest positive integer $k$ such that $a^{k} \equiv 1(\bmod n)$. We denote the order of $a$ modulo $n$ by $\operatorname{Ord}_{n}(a)$. It is easy to see that if $a^{l} \equiv 1(\operatorname{modn})$, then $\operatorname{Ord}_{n}(a) \mid l$. Let $G$ be a finite group with $|G|=p_{1}{ }^{\alpha_{1}} p_{2}{ }^{\alpha_{2}} \ldots p_{k}{ }^{\alpha_{k}}$, where $p_{1}<p_{2}<\ldots<p_{k}$ are prime numbers. We define a directed graph $\gamma(G)$ as follows: the vertex set is $\pi(G)$ and two distinct vertices $p_{i}, p_{j}$ are joined by an edge, denote by $p_{i} \sim p_{j}$, whenever $p_{i} \nsim p_{j}$ in $\Gamma(G)$ and $\operatorname{Ord}_{p_{j}} \alpha_{j}\left(p_{i}\right)>\alpha_{i}$.

The problem of OD-characterizability of simple groups was raised in [2] for the first time. Then many researchers paid attention to characterize finite simple groups by orders and degree patterns of their prime graphs, to mention a few references we will quote [8] and [7].

In this paper we consider the prime graph of a finite group $G$ and prove results which will be used to prove the OD-characterizability of the simple groups $G_{2}(7)$, $B_{3}(5), \mathbb{A}_{11}$, and $\mathbb{A}_{19}$. Of course there are many other simple groups whose ODcharacterizability can be proved using the results of this paper.

If $m$ and $l$ are natural numbers and $p$ is a prime number, the notation $p^{m} \| n$ means that $p^{m} \mid n$ and $p^{m+1} \nmid n$. For a prime number $r$ and a positive integer $n, n_{r}$ denotes the $r$-part of $n$, i.e. type $n_{r}$ is a power of $r$ and $n=m n_{r}$, where $(m, r)=1$.

\section{PRELIMINARIES}

Lemma 1. Let $a>1$ and $n$ be natural numbers and $r$ be a prime number. If $2 \neq r^{n} \| a-1$, then $r^{n+1} \|\left(a^{r}-1\right)$.

Proof. See [3], 3.2.

Lemma 2. Let $p_{i}$ and $p_{j}$ be two distinct prime numbers, $p_{j} \neq 2, \operatorname{Ord}_{p_{j}}\left(p_{i}\right)=m$ and $p_{j} \| p_{i}{ }^{m}-1$, then $\operatorname{Ord}_{p_{j}{ }^{d}}\left(p_{i}\right)=m p_{j}{ }^{d-1}$, where $d$ is a positive integer.

Proof. By Lemma 1 and induction on $t$ we see that

$$
p_{j}{ }^{t} \| p_{i}{ }^{m p_{j}{ }^{t-1}}-1,
$$

where $t$ is an arbitrary natural number. Now we prove the lemma by induction on $d$. If $d=1$, then clearly the lemma holds.

Suppose that $\operatorname{Ord}_{p_{j}{ }^{k}}\left(p_{i}\right)=m p_{j}{ }^{k-1}$. Set $s=\operatorname{Ord}_{p_{j}{ }^{k+1}}\left(p_{i}\right)$. Thus $p_{j}{ }^{k+1} \mid p_{i}{ }^{s}-1$ and so $p_{j}{ }^{k} \mid p_{i}{ }^{s}-1$. Hence $m p_{j}{ }^{k-1} \mid s$, because $\operatorname{Ord}_{p_{j}{ }^{k}}\left(p_{i}\right)=m p_{j}{ }^{k-1}$. On the other hand by (2.1) we have $p_{j}{ }^{k+1} \mid p_{i}{ }^{m p_{j}{ }^{k}}-1$ and since $\operatorname{Ord}_{p_{j}{ }^{k+1}}\left(p_{i}\right)=s, s \mid m p_{j}{ }^{k}$. It follows that $m p_{j}{ }^{k-1}|s| m p_{j}{ }^{k}$. This means that $s=m p_{j}{ }^{k-1}$ or $s=m p_{j}{ }^{k}$. If $s=m p_{j}{ }^{k-1}$, then we have $p_{j}{ }^{k+1} \mid p_{i}{ }^{m p_{j}{ }^{k-1}}-1$. But by (2.1) $p_{j}{ }^{k} \| p_{i}{ }^{m p_{j}{ }^{k-1}}-1$. This contradiction shows that $\operatorname{Ord}_{p_{j}{ }^{k+1}}\left(p_{i}\right)=s=m p_{j}{ }^{k}$. Therefore $\operatorname{Ord}_{p_{j}{ }^{k+1}}\left(p_{i}\right)=m p_{j}{ }^{k}$ and the lemma is proved. 
Lemma 3. Let $G$ be a finite group with $t(G) \geq 2$. If $N \unlhd G$ is a $\pi_{i-\text { group, then }}$ $\left(\prod_{j=1, j \neq i}^{t(G)} m_{j}\right)|| N \mid-1$.

Proof. See Lemma 8 of [1].

Lemma 4. Let $G$ be a finite group with $|G|=p_{1}{ }^{\alpha_{1}} p_{2}{ }^{\alpha_{2}} \ldots p_{n}{ }^{\alpha_{n}}, p_{1}<p_{2}<\ldots<p_{n}$ where $p_{i}$ is a prime number, $1 \leq i \leq n$. Also assume that $M$ is an arbitrary normal subgroup of $G$. Then the following holds:

1) If $p_{i}, p_{j} \in \pi(G)$ and $p_{i} \sim p_{j}$ in $\gamma(G)$, then $p_{i}|| M \mid$ implies that $p_{j}|| M \mid$, where $p_{i}, p_{j}$ are distinct prime numbers.

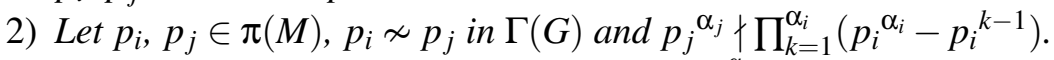
If $\left[\prod_{k=1}^{\alpha_{i}}\left(p_{i}{ }^{\alpha_{i}}-p_{i}{ }^{k-1}\right)\right]_{p_{j}} \mid p_{j}{ }^{\alpha_{j}}$, then $\frac{p_{j}{ }^{\alpha_{j}}}{\left[\prod_{k=1}^{\alpha_{i}}\left(p_{i}{ }^{\alpha_{i}}-p_{i}{ }^{k-1}\right)\right]_{p_{j}}}|| M \mid$.

3) If $p_{i}, p_{j} \in \pi(M), p_{i} \nsim p_{j}$ in $\Gamma(G)$ and $\operatorname{Ord}_{p_{j}{ }^{d}}\left(p_{i}\right)>\alpha_{i}$ for some integer $1 \leq d \leq \alpha_{j}$, then $p_{j}{ }^{\alpha_{j}+1-d}|| M \mid$.

Proof. 1) Since $p_{i} \sim p_{j}$ in $\gamma(G)$, we conclude that $p_{i} \nsim p_{j}$ in $\Gamma(G)$ and $\operatorname{Ord}_{p_{j}} \alpha_{j}\left(p_{i}\right)$ $>\alpha_{i}$. We suppose that $p_{i}|| M \mid$. By Frattini argument $N_{G}\left(M_{p_{i}}\right) M=G$, where $M_{p_{i}}$ is a Sylow $p_{i}$-subgroup of $M$. If $p_{j} \nmid|M|$, then since $p_{j}{ }^{\alpha_{j}}|| G \mid$, we have $p_{j}{ }^{\alpha_{j}}|| N_{G}\left(M_{p_{i}}\right) \mid$ and so $N_{G}\left(M_{p_{i}}\right)$ has a subgroup, say $L$ of order $p_{j}{ }^{\alpha_{j}} . M_{p_{i}} \unlhd N_{G}\left(M_{p_{i}}\right)$ implies that $L M_{p_{i}} \leq N_{G}\left(M_{p_{i}}\right)$. On the other hand there is an positive integer $\beta \leq \alpha_{i}$ such that $\left|L M_{p_{i}}\right|=p_{j}{ }^{\alpha_{j}} p_{i}{ }^{\beta}$ and since $p_{i} \nsim p_{j}$ in $\Gamma(G)$, the prime graph of $L M_{p_{i}}$ is not connected. Also $M_{p_{i}} \unlhd L M_{p_{i}}$. Thus $p_{j}{ }^{\alpha_{j}} \mid p_{i}{ }^{\beta}-1$ by Lemma 3. Hence $\operatorname{Ord}_{p_{j}}{ }^{\alpha_{j}}\left(p_{i}\right) \mid \beta$. In particular we have $\operatorname{Ord}_{p_{j}} \alpha_{j}\left(p_{i}\right) \leq \alpha_{i}$ and this is a contradiction and so $p_{j}|| M \mid$.

2) We have $N_{G}\left(M_{p_{i}}\right) M=G$. Thus $\frac{p_{j}{ }_{j}}{\left|N_{G}\left(M_{p_{i}}\right)\right|_{p_{j}}}|| M \mid$. Moreover if $N$ is a minimal normal subgroup of $N_{G}\left(M_{p_{i}}\right)$ such that $N \leq M_{p_{i}}$, then $N$ is isomorphic to a direct product of cyclic groups $\mathbb{Z}_{p_{i}}$. Assume that $N$ is isomorphic to a direct product of $r$ cyclic group $\mathbb{Z}_{p_{i}} . \quad\left(N \cong \mathbb{Z}_{p_{i}} \times \ldots \times \mathbb{Z}_{p_{i}}\right)$. Since $\frac{N_{G}\left(M_{p_{i}}\right)}{C_{N_{G}\left(M_{p_{i}}\right)}(N)} \hookrightarrow A u t(N)$, we have $\frac{\left|N_{G}\left(M_{p_{i}}\right)\right|}{\left|C_{N_{G}\left(M_{p_{i}}\right)}(N)\right|}|\operatorname{Aut}(N)|=\left|\operatorname{Aut}\left(\mathbb{Z}_{p_{i}}{ }^{r}\right)\right|=\left|G l_{r}\left(p_{i}\right)\right|=\prod_{k=1}^{r}\left(p_{i}{ }^{r}-p_{i}{ }^{k-1}\right)$. This implies that $\left|N_{G}\left(M_{p_{i}}\right)\right||| C_{N_{G}\left(M_{p_{i}}\right)}(N) \mid \prod_{k=1}^{r}\left(p_{i}^{r}-p_{i}^{k-1}\right)$. But since $p_{i} \nsim p_{j}$ in $\Gamma(G), p_{j} \nmid$ $C_{N_{G}\left(M_{p_{i}}\right)}(N) \mid$. (Note that $N$ is a $p_{i}$-group).

Thus $\left|N_{G}\left(M_{p_{i}}\right)\right|_{p_{j}} \mid\left[\prod_{k=1}^{r}\left(p_{i}^{r}-p_{i}{ }^{k-1}\right)\right]_{p_{j}}$. Also since $r \leq \alpha_{i}$,

$$
\left[\prod_{k=1}^{r}\left(p_{i}^{r}-p_{i}^{k-1}\right)\right]_{p_{j}} \mid\left[\prod_{k=1}^{\alpha_{i}}\left(p_{i}^{\alpha_{i}}-p_{i}^{k-1}\right)\right]_{p_{j}}
$$

Therefore $\left|N_{G}\left(M_{p_{i}}\right)\right|_{p_{j}} \mid\left[\prod_{k=1}^{\alpha_{i}}\left(p_{i}^{\alpha_{i}}-p_{i}^{k-1}\right)\right]_{p_{j}}$. 
Now from $N_{G}\left(M_{p_{i}}\right) M=G$, we conclude that $|G|=\left|N_{G}\left(M_{p_{i}}\right) M\right|=\frac{\left|N_{G}\left(M_{p_{i}}\right)\right||M|}{\left|N_{G}\left(M_{p_{i}}\right) \cap M\right|}$ and so $|G||| N_{G}\left(M_{p_{i}}\right)|| M \mid$. Thus $p_{j} \alpha_{j}=\left.|G|_{p_{j}}|| N_{G}\left(M_{p_{i}}\right)\right|_{p_{j}}|M|_{p_{j}}$ and since

$$
\left|N_{G}\left(M_{p_{i}}\right)\right|_{p_{j}}\left|\left[\prod_{k=1}^{\alpha_{i}}\left(p_{i}^{\alpha_{i}}-p_{i}^{k-1}\right)\right]_{p_{j}}, p_{j}{ }^{\alpha_{j}}\right|\left[\prod_{k=1}^{\alpha_{i}}\left(p_{i}^{\alpha_{i}}-p_{i}^{k-1}\right)\right]_{p_{j}}|M|_{p_{j}} .
$$

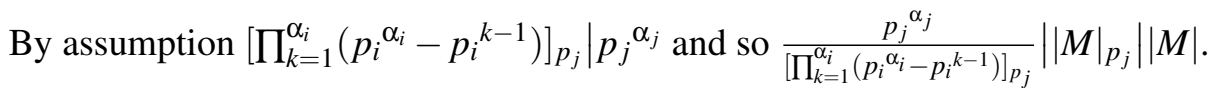

3) We will prove that $p_{j}{ }^{d} \nmid\left|N_{G}\left(M_{p_{i}}\right)\right|$.

If $p_{j}{ }^{d}|| N_{G}\left(M_{p_{i}}\right) \mid$, then $N_{G}\left(M_{p_{i}}\right)$ has a subgroup, say $J$ of order $p_{j}{ }^{d}$.

Since $M_{p_{i}} \unlhd J M_{p_{i}}$ and the prime graph of $J M_{p_{i}}$ is not connected $\left(p_{i} \nsim p_{j}\right.$ in $\left.\Gamma(G)\right)$ by Lemma 3, we have $p_{j}{ }^{d} \mid p_{i}{ }^{e}-1$ for a positive integer $e \leq \alpha_{i}$. It means that $p_{i}{ }^{e} \equiv 1\left(\bmod p_{j}{ }^{d}\right)$. It follows that $\operatorname{Ord}_{p_{j}{ }^{d}}\left(p_{i}\right) \leq \alpha_{i}$, which is a contradiction. Thus $p_{j}{ }^{d} \nmid\left|N_{G}\left(M_{p_{i}}\right)\right|$ and so $\left|N_{G}\left(M_{p_{i}}\right)\right|_{p_{j}} \mid p_{j}{ }^{d-1}$. But since $N_{G}\left(M_{p_{i}}\right) M=G$, we conclude that $|G||| N_{G}\left(M_{p_{i}}\right)|| M \mid$, which implies that $p_{j}{ }^{\alpha_{j}}=\left.\left.|G|_{p_{j}}|| N_{G}\left(M_{p_{i}}\right)\right|_{p_{j}}|M|_{p_{j}}\left|p_{j}{ }^{d-1}\right| M\right|_{p_{j}}$ and so $p_{j}{ }^{\alpha_{j}+1-d}=p_{j}{ }^{\alpha_{j}-(d-1)}|| M \mid$. The proof is completed.

\section{CHARACTERIZATION OF FINITE GROUPS BY PRIME GRAPH AND ORDER OF THE GROUP}

Theorem 1. Let $G$ be a finite group with $|G|=p_{1}{ }^{\alpha_{1}} p_{2}{ }^{\alpha_{2}} \ldots p_{n}{ }^{\alpha_{n}}, p_{1}<p_{2}<\cdots<$ $p_{n}$ where $p_{i}$ is a prime number, $1 \leq i \leq n$. If the directed graph $\gamma(G)$ is strongly connected, then the following assertions hold.

1) There is a simple group $S$ such that $S \unlhd G \leq A u t(S)$ and $\pi(S)=\pi(G)$. Also if $p_{i} \nsim p_{j}$ in $\Gamma(G)$, then $p_{i} \nsim p_{j}$ in $\Gamma(S)$ too and if $p_{i} \sim p_{j}$ in $\Gamma(G)$, then $p_{i} \sim p_{j}$ in $\Gamma(\operatorname{Aut}(S))$ too.

2) Let $p_{i}, p_{j} \in \pi(G), p_{i} \nsim p_{j}$ in $\Gamma(G)$ and $p_{j}{ }^{\alpha_{j}} \nmid \prod_{k=1}^{\alpha_{i}}\left(p_{i}{ }^{\alpha_{i}}-p_{i}{ }^{k-1}\right)$. If $\left[\prod_{k=1}^{\alpha_{i}}\left(p_{i}{ }^{\alpha_{i}}-p_{i}{ }^{k-1}\right)\right]_{p_{j}} \mid p_{j}{ }^{\alpha_{j}}$, then $\frac{p_{j} \alpha_{j}}{\left[\prod_{k=1}^{\alpha_{i}}\left(p_{i}{ }^{\alpha_{i}}-p_{i}{ }^{k-1}\right)\right]_{p_{j}}}|| S \mid$.

3) If $p_{i}, p_{j} \in \pi(G), p_{i} \nsim p_{j}$ in $\Gamma(G)$ and for some integer $1 \leq d \leq \alpha_{j}$, $\operatorname{Ord}_{p_{j} d}\left(p_{i}\right)>\alpha_{i}$, then $p_{j} \alpha^{\alpha+1-d}|| S \mid$.

Proof. Assume that $L$ is a minimal normal subgroup of $G$. Thus $L \neq 1$ and so there is a prime number $p_{i} \in \pi(G)$ such that $p_{i}|| L \mid$. Since $\gamma(G)$ is strongly connected, for all $p_{j} \in \pi(G)$ there exists a directed path from $p_{i}$ to $p_{j}$. So by Lemma 4 and induction on the length of path we can easily see that $p_{j}|| L \mid$ for all $p_{j} \in \pi(G)$. Therefore $\pi(L)=$ $\pi(G)$ and since $\gamma(G)$ is strongly connected, clearly $\Gamma^{c}(G)$ is connected, where $\Gamma^{c}(G)$ denotes the complement of the graph $\Gamma(G)$. Now if $L$ is a direct product of more than one isomorphic simple groups, then since $\pi(L)=\pi(G), \Gamma(G)$ is a complete graph and so $\Gamma(G)^{c}$ is not connected, a contradiction. Hence $L$ is a simple group. On the other hand if for some $q \in \pi(G), q|| C_{G}(L) \mid$, then $q \sim t$ in $\Gamma(G)$ for all $t \in \pi(G)-\{q\}$ and so $\Gamma^{c}(G)$ is not connected, which is contradiction. Thus $C_{G}(L)=1$ and since 
$\frac{G}{C_{G}(L)} \hookrightarrow \operatorname{Aut}(L)$, we conclude that $G \hookrightarrow A u t(L)$. So the proof of Part 1 is completed. We conclude Part 2 and 3 of the Theorem from Lemma 4.

Theorem 2. Let $G$ be a finite group, $|G|=p_{1}{ }^{\alpha_{1}}{p_{2}}^{\alpha_{2}} \ldots p_{n}{ }^{\alpha_{n}}$, where $p_{1}<p_{2}<\cdots<$ $p_{n}$ and $p_{i}$ is a prime number, $1 \leq i \leq n$. If $\gamma_{1}$ is a strongly connected directed subgraph of the graph $\gamma(G)$ and $V_{1}$ is the vertex set of $\gamma_{1}$, then the following assertions hold.

1) There is a simple group $S$ such that $S \unlhd \frac{G}{O_{\pi(G)-V_{1}}(G)} \leq A u t(S), V_{1} \subseteq \pi(S) \subseteq$ $\pi(G)$ and if $p_{i}, p_{j} \in V_{1}$ and $p_{i} \nsim p_{j}$ in $\Gamma(G)$, then $p_{i} \nsim p_{j}$ in $\Gamma(S)$ and if $p_{i} \sim p_{j}$ in $\Gamma(G)$, then $p_{i} \sim p_{j}$ in $\Gamma(A u t(S))\left(O_{\pi(G)-V_{1}}(G)\right.$ is the largest normal subgroup $N$ with $\left.\pi(N)=\pi(G)-V_{1}\right)$.

2) Let $p_{i}, p_{j} \in V_{1}, p_{i} \nsim p_{j}$ in $\Gamma(G)$ and $p_{j}{ }^{\alpha_{j}} \nmid \prod_{\alpha_{j}}^{\alpha_{i}}\left(p_{i}^{\alpha_{i}}-p_{i}^{k-1}\right)$. If $\left[\prod_{k=1}^{\alpha_{i}}\left(p_{i}^{\alpha_{i}}-p_{i}^{k-1}\right)\right]_{p_{j}} \mid p_{j}{ }^{\alpha_{j}}$, then $\frac{p_{j}^{\alpha_{j}}}{\left[\prod_{k=1}^{\alpha_{i}}\left(p_{i}{ }^{\left.\left.\alpha_{i}-p_{i}{ }^{k-1}\right)\right]_{p_{j}}}\right.\right.}|| S \mid$.

3) If $p_{i}, p_{j} \in V_{1}$ and $p_{i} \nsim p_{j}$ in $\Gamma(G)$ and for some integer $1 \leq d \leq \alpha_{j}$, $\operatorname{Ord}_{p_{j} d}\left(p_{i}\right)>\alpha_{i}$, then $p_{j}^{\alpha_{j}+1-d}|| S \mid$.

Proof. Set $L=O_{\pi(G)-V_{1}}(G)$ and $\bar{G}=\frac{G}{L}$. Suppose that $S$ is a minimal normal subgroup of $\bar{G}$. Thus for a normal subgroup of $G$, say $M_{1}$, we have $S=\frac{M_{1}}{L}$, where $L \leq M_{1}$. It is obvious that there is a prime number $q \in V_{1}$, such that $q|| M_{1} \mid$. But there exists a path between $q$ and $t$ for all $t \in V_{1}-\{q\}$. Therefore by Lemma 4 and induction on length we see that $V_{1} \subseteq \pi\left(M_{1}\right)$. It follows that $V_{1} \subseteq \pi(S) \subseteq \pi(G)$. Since $\gamma_{1}$ is a strongly connected subgraph of $\gamma(G)$, for all $p_{i} \in V_{1}$, there exists $p_{j} \in V_{1}$ such that $p_{i} \nsim p_{j}$ in $\Gamma(G)$ and so $S$ is not a direct product of more than one isomorphic simple groups. Hence $S$ is a simple group. Now we prove that $C_{\bar{G}}(S)=1$. Assume that $C_{\bar{G}}(S) \neq 1$. Thus there is a subgroup of $G$, say $K$ such that $C_{\bar{G}}(S)=\frac{K}{L}, L \neq K$. It follows that there is a prime number $r \in V_{1}$ such that $r|| K \mid$. It means that $r|| C_{\bar{G}}(S) \mid$. Moreover since $V_{1} \subseteq \pi(S)$, we conclude that $r \sim t$ in $\Gamma(\bar{G})$ for all $t \in V_{1}-\{r\}$. It is easy to see that $r \sim t$ in $\Gamma(G)$ for all $t \in V_{1}-\{r\}$ and so $r \nsim t$ in $\gamma(G)$, in particular in $\gamma_{1}$ for all $t \in V_{1}-\{r\}$, but this is a contradiction with $\gamma_{1}$ being a strongly connected graph and thus $C_{\bar{G}}(S)=1$. Hence $S \unlhd \bar{G}=\frac{\bar{G}}{1}=\frac{\bar{G}}{C_{\bar{G}}(S)} \leq A u t(S)$.

Now we assume that $p_{i}, p_{j} \in V_{1}$ and $p_{i} \nsim p_{j}$ in $\Gamma(G)$ and $p_{j}^{\alpha_{j}} \nmid \prod_{k=1}^{\alpha_{i}}\left(p_{i}^{\alpha_{i}}-\right.$ $\left.p_{i}{ }^{k-1}\right)$. Also suppose that $\left[\prod_{k=1}^{\alpha_{i}}\left(p_{i}{ }^{\alpha_{i}}-p_{i}{ }^{k-1}\right)\right]_{p_{j}} \mid p_{j}{ }^{\alpha_{j}}$. By using Part 2 of Lemma 4 for $M_{1} \unlhd G$, we conclude that $\frac{p_{j}^{\alpha_{j}}}{\left[\prod_{k=1}^{\alpha_{i}}\left(p_{i}{ }^{\alpha_{i}}-p_{i}^{k-1}\right)\right]_{p_{j}}}|| M_{1} \mid$ and since $p_{j} \in V_{1}, p_{j} \nmid|L|$ and so $\frac{p_{j}^{\alpha_{j}}}{\left[\prod_{k=1}^{\alpha_{i}}\left(p_{i} \alpha_{i}-p_{i}^{k-1}\right)\right]_{p_{j}}}|| \frac{M_{1}}{L}|=| S \mid$, thus the proof of Part 2 is completed.

Similar arguments prove Part 3.

If $p_{i}, p_{j} \in V_{1}, p_{i} \sim p_{j}$ in $\Gamma(S)$, then clearly $p_{i} \sim p_{j}$ in $\Gamma(\bar{G})$ and so in $\Gamma(G)$. Thus if $p_{i} \nsim p_{j}$ in $\Gamma(G)$, then $p_{i} \nsim p_{j}$ in $\Gamma(S)$. Also if $p_{i}, p_{j} \in V_{1}$ and $p_{i} \sim p_{j}$ in $\Gamma(G)$, then there is an element $g \in G$, such that $g^{p_{i} p_{j}}=1$ and $o(g)=p_{i} p_{j}$. Thus $g^{p_{i} p_{j}} \in L$. Since $o(g)=p_{i} p_{j} \nmid|L|, g \notin L$. If $g^{p_{i}} \in L$, then since $\pi(L) \subseteq \pi(G)-V_{1}$ and $p_{i}, p_{j} \in V_{1}$, we conclude that there is a positive integer $m$ such that $\left(p_{i} p_{j}, m\right)=1$ 
and $\left(g^{p_{i}}\right)^{m}=g^{p_{i} m}=1$. This implies that $p_{i} p_{j} \mid p_{i} m$, because $o(g)=p_{i} p_{j}$, thus $p_{j} \mid m$, a contradiction. Therefore $g^{p_{i}} \notin L$. Similarly $g^{p_{j}} \notin L$ and so $o(g L)=p_{i} p_{j}$. Thus $p_{i} \sim p_{j}$ in $\Gamma(\bar{G})$. But since $\bar{G} \leq A u t(S), p_{i} \sim p_{j}$ in $\Gamma(\operatorname{Aut}(S))$ and the proof is completed.

\section{OD-CHARACTERIZABILITY OF FINITE GROUPS}

Let $G$ be a finite group and $|G|=p_{1}{ }^{\alpha_{1}} p_{2}{ }^{\alpha_{2}} \ldots p_{n}{ }^{\alpha_{n}}$, where $p_{1}<p_{2}<\cdots<p_{n}$ and $p_{i}$ is a prime number, $1 \leq i \leq n$. For $i=1,2, \ldots, n$, set $R\left(p_{i}\right)=\mid\left\{p_{j} \in \pi(G) \mid p_{i} \neq p_{j}\right.$, $\operatorname{Ord}_{p_{j}} \alpha_{j}\left(p_{i}\right)>\alpha_{i}$ and $\left.\operatorname{Ord}_{p_{i} \alpha_{i}}\left(p_{j}\right)>\alpha_{j}\right\} \mid$. We have the following three propositions.

Proposition 1. Let $G$ be a finite group with $|G|=p_{1}{ }^{\alpha_{1}} p_{2}{ }^{\alpha_{2}} \ldots p_{n}{ }^{\alpha_{n}}, p_{1}<p_{2}<$ $\cdots<p_{n}$, $p_{i}$ is a prime number, $1 \leq i \leq n$. Assume that there is $p_{m} \in \pi(G)$ such that $\operatorname{deg}\left(p_{m}\right)=0$ in $\Gamma(G)$ and $R\left(p_{m}\right)=n-1$. Then the following assertions hold.

1) There is a simple group $S$ such that $S \unlhd G \leq A u t(S), \pi(S)=\pi(G)$. Also we have $\operatorname{deg}_{\Gamma(S)}\left(p_{i}\right) \leq \operatorname{deg}_{\Gamma(G)}\left(p_{i}\right) \leq \operatorname{deg}_{\Gamma(\operatorname{Aut}(S))}\left(p_{i}\right), 1 \leq i \leq n$.

2) If $p_{l} \in \pi(G), p_{l}{ }^{\alpha_{l}} \nmid \prod_{k=1}^{\alpha_{m}}\left(p_{m}{ }^{\alpha_{m}}-p_{m}{ }^{k-1}\right)$ and $\left[\prod_{k=1}^{\alpha_{m}}\left(p_{m}{ }^{\alpha_{m}}-p_{m}{ }^{k-1}\right)\right] p_{l} \mid p_{l}{ }^{\alpha_{l}}$, then $\frac{p_{l} \alpha_{l}}{\left[\prod_{k=1}^{\alpha_{m}}\left(p_{m}{ }^{\alpha_{m}}-p_{m}{ }^{k-1}\right)\right]_{p_{l}}}|| S \mid$.

3) If $p_{l} \in \pi(G), p_{m}{ }^{\alpha_{m}} \nmid \prod_{k=1}^{\alpha_{l}}\left(p_{l}{ }^{\alpha_{l}}-p_{l}{ }^{k-1}\right)$ and $\left[\prod_{k=1}^{\alpha_{l}}\left(p_{l}{ }^{\alpha_{l}}-p_{l}{ }^{k-1}\right)\right]_{p_{m}} \mid p_{m}{ }^{\alpha_{m}}$, then $\frac{p_{m}{ }^{\alpha_{m}}}{\left[\prod_{k=1}^{\alpha_{l}}\left(p_{l}{ }^{\alpha_{l}}-p_{l}{ }^{k-1}\right)\right]_{p_{m}}}|| S \mid$.

4) If $p_{l} \in \pi(G)$ and $\operatorname{Ord}_{p_{l} d}\left(p_{m}\right)>\alpha_{m}$ for some integer $1 \leq d \leq \alpha_{l}$, then $p_{l}^{\alpha_{l}+1-d}|| S \mid$.

5) If $p_{l} \in \pi(G)$ and $\operatorname{Ord}_{p_{m} d}\left(p_{l}\right)>\alpha_{l}$. for some integer $1 \leq d \leq \alpha_{m}$, then $p_{m}{ }^{\alpha_{m}+1-d}|| S \mid$.

Proof. By Theorem 1 it is sufficient to prove that $\gamma(G)$ is strongly connected. Since $\operatorname{deg}\left(p_{m}\right)=0$ in $\Gamma(G), p_{i} \nsim p_{m}$ in $\Gamma(G)$ for all $i \neq m, 1 \leq i \leq n$ and since $R\left(p_{m}\right)=n-1$, $\operatorname{Ord}_{p_{m} \alpha_{m}}\left(p_{i}\right)>\alpha_{i}$ and $\operatorname{Ord}_{p_{i} \alpha_{i}}\left(p_{m}\right)>\alpha_{m}$ for all $i \neq m, 1 \leq i \leq n$. Hence there is a directed edge from $p_{i}$ to $p_{m}$ and from $p_{m}$ to $p_{i}$ for all $i \neq m, 1 \leq i \leq n$.

Now assume that $p_{a}, p_{b}$ are two arbitrary vertices in $\gamma(G)$. Then by above discussion there is a directed edge from $p_{a}$ to $p_{m}$ and from $p_{m}$ to $p_{a}$ in $\gamma(G)$. Also there is a directed edge from $p_{m}$ to $p_{b}$ and from $p_{b}$ to $p_{m}$. Thus there is a directed path from $p_{a}$ to $p_{b}$. Therefore $\gamma(G)$ is strongly connected.

Since for all $q \in \pi(G)-\left\{p_{m}\right\}, q \nsim p_{m}$ in $\Gamma(G), 2,3,4$ and 5 are concluded from Theorem 1 Part 2 and 3.

Proposition 2. Let $G$ be a finite group with $|G|=p_{1}{ }^{\alpha_{1}} p_{2}{ }^{\alpha_{2}} \ldots p_{n}{ }^{\alpha_{n}}, p_{1}<p_{2}<\cdots<$ $p_{n}$, $p_{i}$ is a prime number, $1 \leq i \leq n$. Assume that there exists $p_{m} \in \pi(G)$ such that $\operatorname{deg}\left(p_{m}\right)=1$ in $\Gamma(G)$ and $R\left(p_{m}\right)=n-1$. Then the following assertions hold.

1) There exists a simple group $S$ and a prime number $p_{r} \in \pi(G)-\left\{p_{m}\right\}$ such that $S \unlhd \frac{G}{O_{p_{r}}(G)} \leq \operatorname{Aut}(S)$ and $\pi(G)-\left\{p_{r}\right\} \subseteq \pi(S) \subseteq \pi(G) . \quad\left(O_{p_{r}}(G)\right.$ is the largest normal subgroup $N$ of $G$ with $\left.\pi(N)=\left\{p_{r}\right\}\right)$. 
2) a) If $p_{s} \in \pi(G)$ and $\operatorname{deg}\left(p_{s}\right)=n-1$ in $\Gamma(G)$, then there is a simple group $S$ such that $S \unlhd \frac{G}{O_{p_{s}}(G)} \leq A u t(S)$ and $\pi(G)-\left\{p_{s}\right\} \subseteq \pi(S) \subseteq \pi(G)$.

b) If $p_{t} \in \pi(G)-\left\{p_{s}, p_{m}\right\}, \quad p_{t}{ }^{\alpha_{t}} \nmid \prod_{k=1}^{\alpha_{m}}\left(p_{m}{ }^{\alpha_{m}}-p_{m}{ }^{k-1}\right) \quad$ and $\left[\prod_{k=1}^{\alpha_{m}}\left(p_{m}{ }^{\alpha_{m}}-p_{m}{ }^{k-1}\right)\right]_{p_{t}} \mid p_{t}{ }^{\alpha_{t}}$, then $\frac{p_{t}{ }^{\alpha_{t}}}{\left[\prod_{k=1}^{\alpha_{m}}\left(p_{m} \alpha_{m}-p_{m}{ }^{k-1}\right)\right]_{p_{t}}}|| S \mid$.

c) If $p_{t} \in \pi(G)-\left\{p_{s}, p_{m}\right\}, \quad p_{m}{ }^{\alpha_{m}} \nmid \prod_{k=1}^{\alpha_{t}}\left(p_{t}{ }^{\alpha_{t}}-p_{t}{ }^{k-1}\right)$ and $\left[\prod_{k=1}^{\alpha_{t}}\left(p_{t}{ }^{\alpha_{t}}-p_{t}{ }^{k-1}\right)\right]_{p_{m}} \mid p_{m}{ }^{\alpha_{m}}$, then $\frac{p_{m} \alpha_{m}}{\left[\prod_{k=1}^{\alpha_{t}}\left(p_{t}^{\alpha_{t}}-p_{t}{ }^{k-1}\right)\right]_{p_{m}}}|| S \mid$.

d) If $p_{t} \in \pi(G)-\left\{p_{s}, p_{m}\right\}$ and $\operatorname{Ord}_{p_{t}{ }^{d}}\left(p_{m}\right)>\alpha_{m}$ for some integer $1 \leq d \leq \alpha_{t}$, then $p_{t}{ }^{\alpha_{t}+1-d}|| S \mid$.

e) If $p_{t} \in \pi(G)-\left\{p_{s}, p_{m}\right\}$ and $\operatorname{Ord}_{p_{m}{ }^{d}}\left(p_{t}\right)>\alpha_{t}$ for some integer $1 \leq d \leq \alpha_{m}$, then $p_{m}{ }^{\alpha_{m}+1-d}|| S \mid$.

Proof. 1) By Theorem 2 it is sufficient to prove that $\gamma(G)$ has a strongly connected subgraph with $n-1$ vertices. Since $\operatorname{deg}\left(p_{m}\right)=1$ in $\Gamma(G)$, there exists $p_{r} \in \pi(G)$ such that $p_{r} \sim p_{m}$ in $\Gamma(G)$. If $p_{i}$ is an arbitrary vertex of the directed graph $\gamma(G)$ such that $p_{i} \neq p_{r}, p_{m}$, then since $R\left(p_{m}\right)=n-1$, we conclude that $\operatorname{Ord}_{p_{i}} \alpha_{i}\left(p_{m}\right)>\alpha_{m}$ and $\operatorname{Ord}_{p_{m} \alpha_{m}}\left(p_{i}\right)>\alpha_{i}$. On the other hand $p_{i} \nsim p_{m}$ in $\Gamma(G)$ and so there is an edge from $p_{i}$ to $p_{m}$ and from $p_{m}$ to $p_{i}$ in $\gamma(G)$.

Now if $p_{a}, p_{b}$ are two arbitrary vertices of $\gamma(G)$ such that $p_{a}, p_{b} \neq p_{r}, p_{m}$, then there is an edge from $p_{a}$ to $p_{m}$ and from $p_{m}$ to $p_{a}$, also from $p_{b}$ to $p_{m}$ and from $p_{m}$ to $p_{b}$ in $\gamma(G)$. Thus there is a path from $p_{a}$ to $p_{b}$. Hence there is a strongly connected subgraph of $\gamma(G)$ such that its vertex set is equal to $\pi(G)-\left\{p_{r}\right\}$. Therefore by Theorem 2, there is a simple group $S$ such that $S \unlhd \frac{G}{O_{p_{r}}(G)} \leq \operatorname{Aut}(S)$ and $\pi(G)-\left\{p_{r}\right\} \subseteq \pi(S) \subseteq \pi(G)$.

2) Assume that there exists $p_{s} \in \pi(G)$ such that $\operatorname{deg}\left(p_{s}\right)=n-1$ in $\Gamma(G)$. So $p_{s}$ is joint to all vertices in $\Gamma(G)$. In particular $p_{s} \sim p_{m}$ in $\Gamma(G)$.

By similar argument as in Part 1 we can see that $\gamma(G)$ has a strongly connected subgraph such that its vertex set is equal to $\pi(G)-\left\{p_{s}\right\}$. Thus by Theorem 2, there is a simple group $S$ such that $S \unlhd \frac{G}{O_{p_{S}}(G)} \leq \operatorname{Aut}(S)$ and $\pi(G)-\left\{p_{s}\right\} \subseteq \pi(S) \subseteq \pi(G)$. Also b, c, d, e are concluded from Theorem 2 Part 2 and 3.

We define $(m)^{*}$ for all $m \in \mathbb{Z}$ by $(m)^{*}=\left\{\begin{array}{cc}m & \text { for } m>0 \\ 0 & \text { for } m \leq 0 .\end{array}\right.$

Proposition 3. Let $G$ be a finite group with $|G|=p_{1}{ }^{\alpha_{1}} p_{2}{ }^{\alpha_{2}} \ldots p_{n}{ }^{\alpha_{n}}, p_{1}<p_{2}<\cdots<$ $p_{n}, p_{i}$ is a prime number, $1 \leq i \leq n$. We set $M=\max \left\{\left(R\left(p_{i}\right)-\operatorname{deg}\left(p_{i}\right)\right)^{*} \mid 1 \leq i \leq n\right\}$ and $m=\min \left\{\left(R\left(p_{i}\right)-\operatorname{deg}\left(p_{i}\right)\right)^{*} \mid 1 \leq i \leq n\right\}$. If $M+m \geq n-1$, then there is a simple group $S$ such that $S \unlhd G \leq A u t(S)$ and $\pi(S)=\pi(G)$. Also $\operatorname{deg}_{\Gamma(S)}(q) \leq \operatorname{deg}_{\Gamma(G)}(q) \leq$ $\operatorname{deg}_{\Gamma(\operatorname{Aut}(S))}(q)$ for all $q \in \pi(G)$.

Proof. By Theorem 1 it is sufficient to prove that $\gamma(G)$ is strongly connected. So assume that $p_{d}$ is an arbitrary vertex of $\gamma(G)$. We define $A_{d}$ and $B_{d}$ as follows:

$$
A_{d}=\left\{p_{i} \in \pi(G) \mid p_{i} \neq p_{d}, p_{i} \nsim p_{d} \text { in } \Gamma(G)\right\},
$$




$$
B_{d}=\left\{p_{j} \in \pi(G) \mid p_{j} \neq p_{d}, \operatorname{Ord}_{p_{j}} \alpha_{j}\left(p_{d}\right)>\alpha_{d} \text { and } \operatorname{Ord}_{p_{d} \alpha_{d}}\left(p_{j}\right)>\alpha_{j}\right\} .
$$

Thus $\left|A_{d}\right|=n-1-\operatorname{deg}\left(p_{d}\right),\left|B_{d}\right|=R\left(p_{d}\right)$, where $\operatorname{deg}\left(p_{d}\right)$ is the degree of $p_{d}$ in $\Gamma(G)$.

Moreover $A_{d} \cap B_{d}$ is equal to set of all vertices in $\gamma(G)$ that are joined to $p_{d}$ and also $p_{d}$ is joined to them by an edge. Since $p_{d} \notin A_{d} \cup B_{d}, A_{d} \cup B_{d} \subseteq \pi(G)-\left\{p_{d}\right\}$ and so $\left|A_{d} \cup B_{d}\right| \leq n-1$. Therefore we have $\left|A_{d} \cup B_{d}\right|=n-1-\operatorname{deg}\left(p_{d}\right)+R\left(p_{d}\right)-$ $\left|A_{d} \cap B_{d}\right| \leq n-1$. Hence $\left|A_{d} \cap B_{d}\right| \geq R\left(p_{d}\right)-\operatorname{deg}\left(p_{d}\right)$. Since $\left|A_{d} \cap B_{d}\right| \geq 0$, we have $\left|A_{d} \cap B_{d}\right| \geq\left(R\left(p_{d}\right)-\operatorname{deg}\left(p_{d}\right)\right)^{*}$.

But $\left(R\left(p_{d}\right)-\operatorname{deg}\left(p_{d}\right)\right)^{*} \geq m$, which implies that $\left|A_{d} \cap B_{d}\right| \geq m$. Thus there exist $m$ vertices in $\gamma(G)$ that are joined to $p_{d}$ and also $p_{d}$ is joined to them by an edge, where $p_{d}$ is an arbitrary vertex of $\gamma(G)$. Denote the set of all these $m$ vertices by $E_{d}$.

Now we assume that $p_{c} \in \pi(G)$ and $M=\left(R\left(p_{c}\right)-\operatorname{deg}\left(p_{c}\right)\right)^{*}$. Then by a similar argument we see that there exist $M$ vertices in $\gamma(G)$ that are joined to $p_{c}$ and also $p_{c}$ is joined to them by an edge. Denote the set of all these $M$ vertices by $F_{c}$.

We will show that if $p_{u} \in \pi(G)$ is different from $p_{c}$, then there is a directed path from $p_{u}$ to $p_{c}$ and from $p_{c}$ to $p_{u}$. Since $p_{u} \neq p_{c}, p_{u} \nsim p_{c}$ and $p_{c} \nsim p_{u}$ in $\gamma(G)$. We know that $p_{u} \sim q$ and $q \sim p_{u}$ in $\gamma(G)$ for all $q \in E_{u}$. If $E_{u} \cap F_{c}=\varnothing$, then since $\left\{p_{u}\right\} \cup E_{u} \cup\left\{p_{c}\right\} \cup F_{c} \subseteq \pi(G), p_{u} \neq p_{c}, p_{u} \nsim p_{c}$ and $p_{c} \nsim p_{u}$ in $\gamma(G)$, we have $\left|\left\{p_{u}\right\} \cup E_{u} \cup\left\{p_{c}\right\} \cup F_{c}\right|=1+m+1+M \leq n$, which is a contradiction with assumption, $(M+m \geq n-1)$. Thus $E_{u} \cap F_{c} \neq \varnothing$. Suppose that $p_{v} \in E_{u} \cap F_{c}$. It follows that $p_{u} \sim p_{v}, p_{v} \sim p_{u}, p_{c} \sim p_{v}$ and $p_{v} \sim p_{c}$. Hence $p_{u} \rightarrow p_{v} \rightarrow p_{c}$ is a directed path from $p_{u}$ to $p_{c}$ and $p_{c} \rightarrow p_{v} \rightarrow p_{u}$ is a directed path from $p_{c}$ to $p_{u}$. So we proved that for all $p_{u} \in \pi(G)$ there exists a directed path from $p_{u}$ to $p_{c}$ and there is a directed path from $p_{c}$ to $p_{u}$.

Now we assume that $p_{a}, p_{b}$ are two arbitrary vertices of $\gamma(G)$. Thus by the above discussion there is a path from $p_{c}$ to $p_{a}$ and from $p_{a}$ to $p_{c}$, also there is a path from $p_{c}$ to $p_{b}$ and from $p_{b}$ to $p_{c}$. Therefore there is a path from $p_{a}$ to $p_{b}$ and so $\gamma(G)$ is strongly connected and the proof is completed.

\section{APPLICATIONS}

We give some examples of characterization of finite groups by prime graph and OD-characterization of them.

We note that the following examples are proved in [4] and a few more papers. But our proofs are based on Theorems 1 and 2 and Propositions 1, 2 and 3. The prime graphs of all groups considered are obtained by [6].

Example 1. We consider the simple group $C_{2}(7)$. We know that $\left|C_{2}(7)\right|=$ $2^{8} \cdot 3^{2} \cdot 5^{2} \cdot 7^{4}$ and $2 \sim 3,2 \sim 7,3 \sim 7,5 \nsim 2,5 \nsim 7$ and $5 \nsim 3$ in $\Gamma\left(C_{2}(7)\right)$. Since $\operatorname{Ord}_{5^{2}}(2)>8, \operatorname{Ord}_{5^{2}}(3)>2, \operatorname{Ord}_{2^{8}}(5)>2, \operatorname{Ord}_{3^{2}}(5)>2$, we deduce that $E_{\gamma\left(C_{2}(7)\right)} \supseteq$ $\{(2,5),(5,2),(3,5),(5,3)\}$, where $E_{\gamma\left(C_{2}(7)\right)}$ is the edge set of $\gamma(G)$. Hence there exists a strongly connected subgraph of $\gamma\left(C_{2}(7)\right)$ that its vertex set is $\{2,3,5\}$. 
Now if $G$ is a finite group with $|G|=\left|C_{2}(7)\right|$ and $\Gamma(G)=\Gamma\left(C_{2}(7)\right)$, then $\gamma(G)=$ $\gamma\left(C_{2}(7)\right)$ and so there exists a strongly connected subgraph of $\gamma(G)$ that its vertex set is $\{2,3,5\}$. Thus by Theorem 2 there is a simple group $S$ such that $S \unlhd \frac{G}{O_{7}(G)} \leq \operatorname{Aut}(S)$ and $\{2,3,5\} \subseteq \pi(S) \subseteq \pi(G)=\{2,3,5,7\}$. Since $3 \nsim 5$ in $\Gamma\left(C_{2}(7)\right)=\Gamma(G)$ and $\operatorname{Ord}_{5}(3)>2$ by Part 3 of Theorem 2, we conclude that $5^{2+1-1}=5^{2}|| S \mid$. Similarly since $2 \nsim 5$ in $\Gamma(G)$ and $\operatorname{Ord}_{2^{4}}(5)>2,2^{8+1-4}=2^{5}|| S \mid$. Now by Table 4 of [5] we see that $S \cong B_{2}(7)$ or $S \cong C_{2}(7)$ and since $S \leq \frac{G}{O_{7}(G)}$ and $\frac{|G|}{\left|O_{7}(G)\right|}|G|=\left|C_{2}(7)\right|$, we conclude that $O_{7}(G)=1$ and $G=S$ and so $G \cong B_{2}(7)$ or $G \cong C_{2}(7)$.

Hence if $\Gamma(G)=\Gamma\left(C_{2}(7)\right)$ and $|G|=\left|C_{2}(7)\right|$, then $G \cong C_{2}(7)$ or $G \cong B_{2}(7)$.

Example 2. We consider the simple group $B_{3}(5)$. We know that $\left|B_{3}(5)\right|=$ $2^{9} \cdot 3^{4} \cdot 5^{9} \cdot 7 \cdot 13 \cdot 31$ and $2 \sim 3,2 \sim 5,2 \sim 132 \sim 31,3 \sim 5,3 \sim 7,3 \sim 13$ and $5 \sim 13$ in $\Gamma\left(B_{3}(5)\right)$ and $7 \nsim i, 31 \nsim j$ for $i \in\{2,5,13,31\}$ and $j \in\{3,5,7,13\}$ in $\Gamma(G)$. We have $\operatorname{Ord}_{31}(3)>4, \operatorname{Ord}_{3^{4}}(31)>1, \operatorname{Ord}_{7}(31)>1, \operatorname{Ord}_{31}(7)>1, \operatorname{Ord}_{31}(13)>1$ and $\operatorname{Ord}_{13}(31)>1$. Thus $E_{\gamma\left(B_{3}(5)\right)} \supseteq\{(31,3),(3,31),(31,7),(7,31),(31,13),(13,31)\}$, where $E_{\gamma\left(B_{3}(5)\right)}$ is the edge set of $\gamma\left(B_{3}(5)\right)$. Therefore there exists a strongly connected subgraph of $\gamma\left(B_{3}(5)\right)$ that its vertex set is $\{3,7,13,31\}$. Now if $G$ is a finite group with $|G|=\left|B_{3}(5)\right|$ and $\Gamma(G)=\Gamma\left(B_{3}(5)\right)$, then $\gamma(G)=\gamma\left(B_{3}(5)\right)$ and so there exists a strongly connected subgraph of $\gamma(G)$ that its vertex set is $\{3,7,13,31\}$ Thus by Theorem 2, there is a simple group $S$ such that $S \unlhd \frac{G}{O_{\{2,5\}}(G)} \leq \operatorname{Aut}(S)$ and $\{3,7,13,31\} \subseteq \pi(S) \subseteq\{2,3,5,7,13,31\}$. But since $3 \nsim 31$ in $\Gamma(G)=\Gamma\left(B_{3}(5)\right)$ and $3^{4} \nmid 31-1$ by Theorem 2 Part 2 we have $\frac{3^{4}}{[31-1]_{3}}=3^{3}|| S \mid$ and so $3^{3} \cdot 7 \cdot 13 \cdot 31|| S \mid$. Now by Table 4 of [5], we conclude that $S \cong B_{3}(5)$ or $S \cong C_{3}(5)$. Thus $O_{\{2,5\}}(G)=1$ and since $|G|=\left|B_{3}(5)\right|$, we conclude that $G \cong B_{3}(5)$ or $G \cong C_{3}(5)$.

Hence if $\Gamma(G)=\Gamma\left(B_{3}(5)\right)$ and $|G|=\left|B_{3}(5)\right|$, then $G \cong B_{3}(5)$ or $G \cong C_{3}(5)$.

Example 3. We consider the simple group $\mathbb{A}_{11}$. We know that $\left|\mathbb{A}_{11}\right|=2^{7} \cdot 3^{4} \cdot 5^{2}$. $7 \cdot 11$. We can easily see that $\operatorname{deg}(11)=0$ in $\Gamma\left(\mathbb{A}_{11}\right)$. Assume that $G$ is a finite group with $D(G)=D\left(\mathbb{A}_{11}\right)$ and $|G|=\left|\mathbb{A}_{11}\right|$.

Since $\operatorname{Ord}_{11}(2)>7, \operatorname{Ord}_{11}(3)>4, \operatorname{Ord}_{11}(5)>2, \operatorname{Ord}_{11}(7)>1, \operatorname{Ord}_{2^{7}}(11)>1$, $\operatorname{Ord}_{3^{4}}(11)>1, \operatorname{Ord}_{5^{2}}(11)>1$ and $\operatorname{Ord}_{7}(11)>1$, we conclude that $R(11)=4$ and since $|\pi(G)|=5$ by Proposition 1 there is a simple group $S$ such that $S \unlhd G \leq A u t(S)$ and $\pi(S)=\pi(G)=\{2,3,5,7,11\}$. Since $2^{7} \nmid 11-1=10$ and $[10]_{2} \mid 2^{7}$ by Part 2 of Proposition 1 we have $\frac{2^{7}}{[10]_{2}}=2^{6}|| S \mid$. Similarly $3^{4}|| S \mid$. Thus $|S|=2^{a} \cdot 3^{4} \cdot 5^{b} \cdot 7 \cdot 11$, where $6 \leq a \leq 7,1 \leq b \leq 2$ and so by Table 4 of [5] $S$ is isomorphic to $\mathbb{A}_{11}$ and since $S \leq G,|G|=\left|\mathbb{A}_{11}\right|$, we conclude that $G \cong \mathbb{A}_{11}$.

Hence $\mathbb{A}_{11}$ is OD-characterizable.

Example 4. We consider the simple group $\mathbb{A}_{19}$. We know that $\left|\mathbb{A}_{19}\right|=2^{15} \cdot 3^{8} \cdot 5^{3}$. $7^{2} \cdot 11 \cdot 13 \cdot 17 \cdot 19$. Obviously $\operatorname{deg}(19)=0$ in $\Gamma\left(\mathbb{A}_{19}\right)$. Now assume that $G$ is a finite group with $D(G)=D\left(\mathbb{A}_{19}\right)$ and $|G|=\left|\mathbb{A}_{19}\right|$. 
We have $\operatorname{Ord}_{19}(2)>15, \operatorname{Ord}_{19}(3)>8, \operatorname{Ord}_{19}(5)>3, \operatorname{Ord}_{19}(7)>2, \operatorname{Ord}_{19}(11)>$ $1, \operatorname{Ord}_{19}(13)>1, \operatorname{Ord}_{19}(17)>1, \operatorname{Ord}_{2^{15}}(19)>1, \operatorname{Ord}_{3^{8}}(19)>1, \operatorname{Ord}_{5^{3}}(19)>1$, $\operatorname{Ord}_{7^{2}}(19)>1, \operatorname{Ord}_{11}(19)>1, \operatorname{Ord}_{13}(19)>1$ and $\operatorname{Ord}_{17}(19)>1$, thus $R(19)=7$ and since $|\pi(G)|=8$ by Proposition 1 there is a simple group $S$ such that $S \unlhd G \leq$ $\operatorname{Aut}(S)$ and $\pi(S)=\pi(G)=\{2,3,5,7,11,13,17,19\}$. Since $2^{15} \nmid 19-1=18,[18]_{2} \mid 2^{15}$ by Part 2 of Proposition 1 we have $\frac{2^{15}}{[18]_{2}}=2^{14}|| S \mid$. Similarly $\frac{3^{8}}{[18]_{3}}=3^{6}|| S\left|, 5^{3}\right||S|$ and $7^{2}|| S \mid$. Thus $|S|=2^{a} \cdot 3^{b} \cdot 5^{3} \cdot 7^{2} \cdot 11 \cdot 13 \cdot 17 \cdot 19$, where $14 \leq a \leq 15,6 \leq b \leq 8$ and so by Table 4 of $[5] S \cong \mathbb{A}_{19}$ and since $S \leq G,|G|=\left|\mathbb{A}_{19}\right|$, we conclude that $G \cong \mathbb{A}_{19}$.

Hence $\mathbb{A}_{19}$ is OD-characterizable.

\section{ACKNOWLEDGEMENT}

The authors would like to thank the referee for useful suggestions that improved the quality of the paper.

\section{REFERENCES}

[1] G. Chen, "Further reflections on Thompson's conjecture," Journal of Algebra - J ALGEBRA, vol. 218, pp. 276-285, 1999.

[2] M. Darafsheh, A. Moghaddamfar, and A. Zokayi, "A characterization of finite simple groups by the degrees of vertices of their prime graphs," Algebra Colloquium, vol. 12, pp. 431-442, 2005.

[3] C. Hering, "Transitive linear groups and linear groups which contain irreducible subgroups of prime order," Geometriae Dedicata, vol. 2, no. 4, pp. 425-460, 1974, doi: 10.1007/BF00147570.

[4] A. Iranmanesh and B. Khosravi, "A characterization of $C_{2}(q)$ where $q>5$," Commentationes Mathematicae Universitatis Carolinae, vol. 43, pp. 9-21, 2002.

[5] A. Moghaddamfar and S. , "More on the od-characterizability of a finite group," Algebra Colloquium, vol. 18, no. 4, pp. 663-674, 2012.

[6] A. Vasiliev and E. Vdovin, "An adjacency criterion for the prime graph of a finite simple group," Algebra i Logika, vol. 6, pp. 381-406, 2005.

[7] L. Zhang and W. Shi, "Od-characterization of the projective special linear groups $L_{2}(q)$," Algebra Colloquium, vol. 19, no. 03, pp. 509-524, 2012, doi: 10.1142/S1005386712000375.

[8] L. Zhang, W. Shi, C. Shao, and L. Wang, "Od-characterization of the simple group $L_{3}(9)$," Journal of Guangxi University. Natural Science Edition, vol. 34, pp. 120-122, 2009.

Authors' addresses

\section{Ghorbani}

Mazandaran University of Science and Technology, P.O.Box 11111, Behshahr, Iran

E-mail address: m_ghorbanieiust.ac.ir

\section{R. Darafsheh}

School of Mathematics, Statistics and Computer Science, College of Science, University of Tehran, Tehran, Iran

E-mail address: darafsheh@ut.ac.ir

\section{Pedram Yousefzadeh}

Department of Mathematics, K.N. Toosi University of Technology, Tehran, Iran

E-mail address: pedram_yous@yahoo.com 